\title{
Total Internal Reflection of Orbital Angular Momentum Beams
}

\author{
W. Löffler \\ Huygens Laboratory, Leiden University, P.O. Box 9504, 2300 RA Leiden, The Netherlands \\ E-mail: loeffler@physics.leidenuniv.nl \\ N. Hermosa \\ ICFO-Institut de Ciencies Fotoniques, Mediterranean Technology Park, 08860 Castelldefels \\ (Barcelona), Spain \\ Andrea Aiello \\ Max Planck Institute for the Science of Light, Günther-Scharowsky-Straße 1/Bldg. 24, 91058 \\ Erlangen, Germany and Institute for Optics, Information and Photonics, Universität \\ Erlangen-Nürnberg, Staudtstr. 7/B2, 91058 Erlangen

\section{J. P. Woerdman} \\ Huygens Laboratory, Leiden University, P.O. Box 9504, 2300 RA Leiden, The Netherlands

\begin{abstract}
We investigate how beams with orbital angular momentum (OAM) behave under total internal reflection. This is studied in two complementary experiments: In the first experiment, we study geometric shifts of OAM beams upon total internal reflection (GoosHänchen and Imbert-Fedorov shifts, for each the spatial and angular variant), and in the second experiment we determine changes in the OAM mode spectrum of a beam, again upon total internal reflection. As a result we find that in the first case, the shifts are independent of OAM and beam focussing, while in the second case, modifications in the OAM spectrum occur which depend on the input OAM mode as well as on the beam focussing. This is investigated by experiment and theory. We also show how the two methods, beam shifts on the one hand, and OAM spectrum changes on the other, are related theoretically.
\end{abstract}

Keywords: orbital angular momentum, beam shifts

PACS numbers: 41.20.Jb, 42.50.Tx, 42.25.Gy

AMS classification scheme numbers: 


\section{Introduction}

Simple optical reflection at planar interfaces still offers new surprises. More than 60 years ago, Goos and Hänchen found that a realistic optical beam experiences an in-plane displacement with respect to the geometric optics expected path, under total internal reflection (TIR) [1]. This is caused by the fact that each of the plane-wave components, which constitute the beam, picks up a slightly different reflection coefficient. In other words, even if a beam has a flat wavefront (which is approximately true for well-collimated beams), beams are not plane waves and have a finite wave vector spread, which needs to be taken into account during reflection. Nevertheless, beams are the best approximation of a geometric-optics ray, and it turns out that beam shifts are the first-order diffractive corrections to geometric optics. Beam shifts are polarization dependent. In-plane GoosHänchen shifts appear for $p$ and $s$ linear polarization while the (transverse) Imbert-Fedorov shifts appear for circular polarization [2, 3. In recent years, the study of beam shift phenomena got a strong boost by (amongst other effects) the discovery of the Spin-Hall Effect of light [4-6] and angular shifts [7-9]. In the meantime, shifts have been discovered not only for light, but also for matter waves [10, 11].

Beam shifts occurring under partial (external) reflection, such as angular beam shifts [9], are known to be sensitive to the spatial structure of the beam [12. In particular, these shifts are sensitive to the beam's orbital angular momentum [13, which is appearing, for instance, in Laguerre-Gauss (LG) laser modes. We have recently shown that one can describe the diffractive corrections appearing upon reflection also as a change on the transverse mode spectrum of the beam; notably a pure LG input mode was found to acquire sidebands 14 upon external reflection. These results were obtained by external reflection of the input beam. How does this change if we investigate total internal reflection? Specifically, how does the orbital angular momentum influence beam shifts, and how is the OAM spectrum modified by TIR? This has not been studied yet, we shed light on this by theory and experiment.

\section{Shifts of beams with OAM under total internal reflection}

We start with the general case of optical reflection, i.e. we do not yet specialize to the TIR case. Consider an incoming paraxial, monochromatic and homogeneously polarized $(\lambda=1,2 \equiv p, s)$, but otherwise arbitrary, optical field $\mathbf{U}^{i}(x, y, z)=\sum_{\lambda} U^{i}(x, y, z) a_{\lambda} \hat{\mathbf{x}}_{\lambda}^{i}$ propagating along $\hat{\mathbf{x}}_{3}^{i}(z$ coordinate), where $\left(a_{1}, a_{2}\right)$ is the polarization Jones vector of the incoming beam. We use dimensionless quantities in units of $1 / k_{0}$, where $k_{0}$ is the wavevector. The coordinate systems and their unit vectors $\hat{\mathbf{x}}_{\lambda}^{i, r}$ are attached to the incoming $(i)$ and reflected $(r)$ beam, respectively. After reflection at a dielectric interface, the polarization and spatial degree of freedom are coupled by the Fresnel coefficients $r_{p, s}$ as [12]

$$
\mathbf{U}^{r}(x, y, z)=\sum_{\lambda} a_{\lambda} r_{\lambda} U\left(-x+X_{\lambda}, y-Y_{\lambda}, z\right) \hat{\mathbf{x}}_{\lambda}^{r} \equiv \sum_{\lambda} a_{\lambda} r_{\lambda} \mathbf{U}_{\lambda}^{r} .
$$

$X_{\lambda}$ and $Y_{\lambda}$ are the polarization-dependent dimensionless beam shifts:

$$
\begin{aligned}
& X_{1}=-i \partial_{\theta}\left[\ln r_{1}(\theta)\right], Y_{1}=i \frac{a_{2}}{a_{1}}\left(1+\frac{r_{2}}{r_{1}}\right) \cot \theta \\
& X_{2}=-i \partial_{\theta}\left[\ln r_{2}(\theta)\right], Y_{2}=-i \frac{a_{1}}{a_{2}}\left(1+\frac{r_{1}}{r_{2}}\right) \cot \theta
\end{aligned}
$$




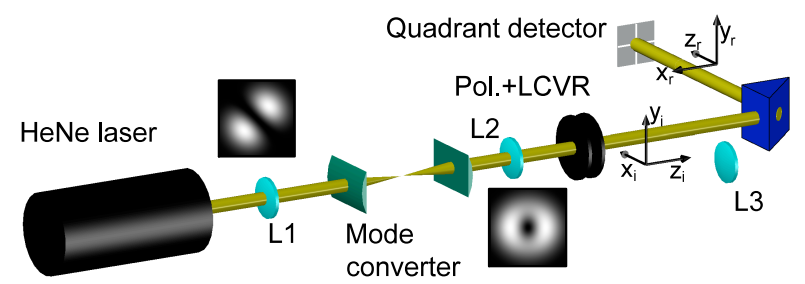

Figure 1. Setup to measure beam shifts under total internal reflection. A HeNe laser with an intra-cavity wire is set to produce a clean $H G_{n 0}$ mode, and an astigmatic mode converter is used to convert this mode in a pure Laguerre-Gauss mode. We modulate the polarization using a variable retarder. The light is then reflected internally using the prism; and for increasing the beam spread $\theta_{0}$ (focussing), lens L3 can be introduced. The polarization-differential reflected beam position is determined using a quadrant detector in combination with a lock-in amplifier.

Their real parts yield the spatial beam shifts, and their imaginary parts the angular beam shifts. They can appear as longitudinal Goos-Hänchen type shifts [1] $X_{\lambda}$ (along $\hat{x}$ coordinate), or as transverse Imbert-Fedorov type shifts $\underline{3}, 4, Y_{\lambda}$ along $\hat{y}$. Transverse shifts $Y_{\lambda}$ require that both $a_{1}$ and $a_{2}$ are finite, such as present in circularly polarized light; this is not necessary for the longitudinal shifts $X_{\lambda}$. To make the step from these dimensionless shifts to observable shifts, we have to find the centroid of the reflected beam:

$$
\langle\mathbf{R}\rangle(z)=\sum_{\lambda} w_{\lambda} \frac{\int \mathbf{R}\left|U\left(-x+X_{\lambda}, y-Y_{\lambda}, z\right)\right|^{2} \mathrm{~d} x \mathrm{~d} y}{\int\left|U\left(-x+X_{\lambda}, y-Y_{\lambda}, z\right)\right|^{2} \mathrm{~d} x \mathrm{~d} y},
$$

where $w_{\lambda}=\left|r_{\lambda} a_{\lambda}\right|^{2} / \sum_{\nu}\left|r_{\nu} a_{\nu}\right|^{2}$ is the fraction of the reflected intensity with polarization $\lambda$, and $\mathbf{R}=x \hat{\mathbf{x}}_{1}^{r}+y \hat{\mathbf{x}}_{2}^{r}$. The shift of the centroid depends on the structure of the field, while the dimensionless beam shifts (Eq. 2) are independent of the exact form of $U$. Eq. 3 can be calculated by Taylor expansion around zero shift $\left(X_{\lambda}=Y_{\lambda}=0\right)$. We obtain for the centroid, which is the expectation value for the $2 \mathrm{D}$ position vector $\mathbf{R}$ at distance $z$ from the beam waist

$$
\langle\mathbf{R}\rangle(z)=\sum_{\lambda} w_{\lambda}\left[\operatorname{Re}\left(\begin{array}{c}
X_{\lambda} \\
Y_{\lambda}
\end{array}\right)+M(z) \operatorname{Im}\left(\begin{array}{c}
X_{\lambda} \\
Y_{\lambda}
\end{array}\right)\right]
$$

where $M(z)$ is a polarization-independent $2 \times 2$ matrix, which depends on the transverse mode of the field [15]. The $z$-dependent diagonal elements of $M(z)$ describe how angular shifts influence the apparent position $\langle\mathbf{R}\rangle(z)$, and the off-diagonal elements effectively mix transverse angular shift into the longitudinal spatial shift, and the longitudinal angular shift into the transverse spatial shift. Evaluating Eq. 3 for Laguerre-Gauss beams with $p=0$ and an OAM of $\ell \hbar$, and using the dimensionless Rayleigh range $\Lambda=2 / \theta_{0}^{2}$, we obtain [12]:

$$
\langle\mathbf{R}\rangle(z)=\sum_{\lambda} w_{\lambda}\left[\operatorname{Re}\left(\begin{array}{c}
X_{\lambda} \\
Y_{\lambda}
\end{array}\right)+\left(\begin{array}{cc}
z / \Lambda & -\ell \\
\ell & z / \Lambda
\end{array}\right) \operatorname{Im}\left(\begin{array}{c}
X_{\lambda} \\
Y_{\lambda}
\end{array}\right)\right]
$$

Finally, we specialize to the case of total internal reflection, where $X_{\lambda}$ and $Y_{\lambda}$ (Eqs. 2 are real, which means that mixing via $M(z)$ does not occur; therefore the shift is expected to be of purely spatial nature and independent of the orbital angular momentum $\ell$. 

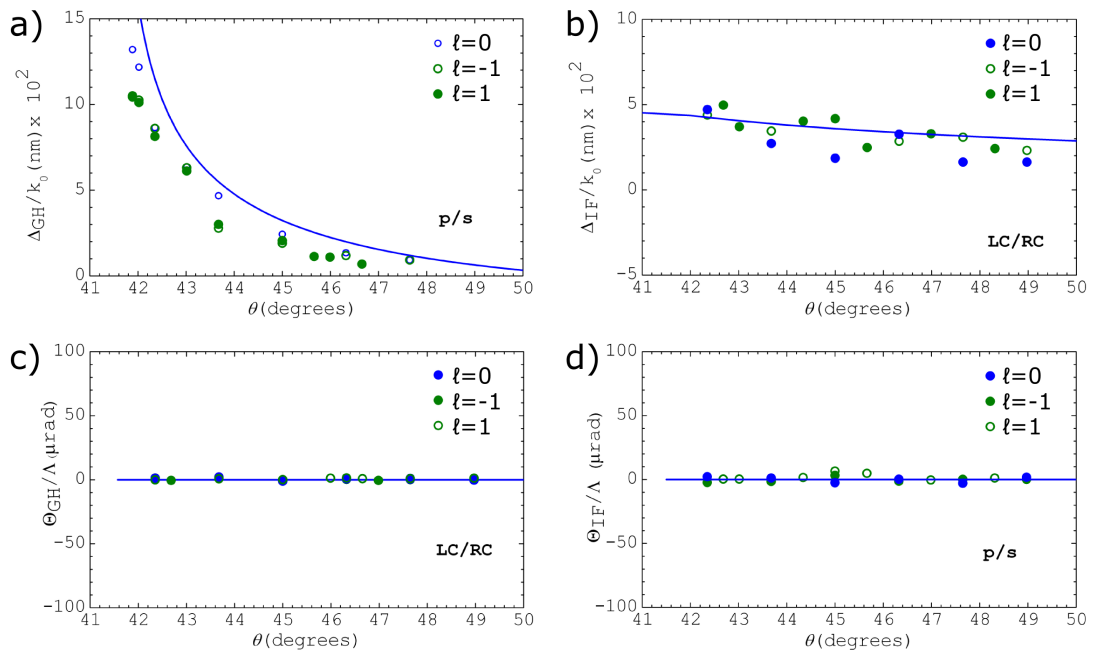

Figure 2. Polarization-differential beam shift measurements for total internal reflection: We see that the spatial Goos-Hänchen shift $\Delta_{G H}$ (a) and Imbert-Fedorov shift $\Delta_{I F}$ (b) are present, but independent on the orbital angular momentum $\ell$ of the beam. The angular Goos-Hänchen shift $\Theta_{G H}$ (c) and angular Imbert-Fedorov shift $\Theta_{I F}$ (d) shifts do not occur. Shown are the real non-dimensionless quantities as measured in the lab.

This brings us to our first experiment as shown in Fig. 1, where we investigate if the OAM mode influences spatial Goos-Hänchen and Imbert-Fedorov shifts under TIR, and if angular shifts disappear as expected. The OAM beam is prepared with a custom HeNe laser and mode conversion. In the laser cavity, a wire (40 $\mu$ m diameter) is introduced, to enforce a Hermite-Gaussian $\left(H G_{n m}\right)$ fundamental mode with $m=0$. This mode is sent through an astigmatic mode converter [13, consisting of two cylindrical lenses. The nodal line of the $\mathrm{HG}$ mode is oriented at $45^{\circ}$ relative to the common axis of the mode converter, such that a $H G_{n 0}$ mode is transformed into the $L G_{\ell p}$ mode with $\ell=n$ and $p=0$. Additional lenses (L1 and L2) are used to ensure mode matching; the final beam after L2 has a beam waist of $775 \mu \mathrm{m}$. After polarization modulation with a liquid-crystal variable retarder (LCVR), this beam is then reflected internally at the hypothenuse of a $45^{\circ}-90^{\circ}-45^{\circ}$ BK7 prism. We measure the polarization-differential beam displacement using a quadrant detector (which is binned to act effectively as a split detector), and lock-in techniques (for details, see [12]). Fig. 2 demonstrates that our theoretical expectations were accurate: Under TIR, only spatial shifts, here in the polarization differential form $\Delta_{G H} \equiv \operatorname{Re}\left[X_{1}-X_{2}\right]$ and $\Delta_{I F} \equiv \operatorname{Re}\left[Y_{1}-Y_{2}\right]$ occur, and are independent of the OAM $\ell$. The angular shifts $\Theta_{G H} \equiv \operatorname{Im}\left[X_{1}-X_{2}\right]$ and $\Theta_{I F} \equiv \operatorname{Im}\left[Y_{1}-Y_{2}\right]$ are identical to zero. The shifts are also independent of the collimation properties of the beam as determined by $\theta_{0}$ (data not shown), which we expect from the discussion above.

\section{Appearance of OAM sidebands under TIR}

We now come to the second experiment which addresses changes in the OAM spectrum upon total internal reflection, following [14]. We start by evaluating Eq. 1 now directly. A first-order Taylor 
expansion around zero shift $\left(X_{\lambda}=Y_{\lambda}=0\right)$ results in

$$
U\left(-x+X_{\lambda}, y-Y_{\lambda}, z\right) \simeq U(-x, y, z)+\mathbf{R}_{\lambda} \cdot \frac{\partial}{\partial \mathbf{R}} U(-x, y, z),
$$

with $\mathbf{R}=(x, y)$ and $\mathbf{R}_{\lambda}=\left(X_{\lambda}, Y_{\lambda}\right)$. We substitute $U$ for the well-known normalized Laguerre-Gauss functions, $U \rightarrow U_{p, \ell} \rightarrow L G_{p}^{\ell}$, where $\ell$ and $p$ are the azimuthal and radial mode indices, respectively. The spatial Fresnel coefficients $c_{\ell, \ell^{\prime}, p, p^{\prime}}^{\lambda}$ describe the scattering amplitude for an incoming $L G_{p}^{\ell}$ mode into the $L G_{p^{\prime}}^{\ell^{\prime}}$ output channel. These coefficients are obtained by OAM decomposition of the shifted reflected beam from Eq. 6

$$
c_{\ell, \ell^{\prime}, p, p^{\prime}}^{\lambda}=\int \mathrm{d}^{2} R L G_{p^{\prime}}^{\ell^{\prime} *}(\mathbf{R}) U_{p, \ell}\left(-x+X_{\lambda}, y-Y_{\lambda}, z\right) .
$$

If we consider only the OAM part of the Laguerre-Gauss modes (by setting $p=p^{\prime}=0$ ), we find the following simple coefficients (upper and lower signs refer to the case $\ell \geqslant 0$, and $\ell<0$, respectively):

$$
c_{\ell, \ell^{\prime}}^{\lambda}= \begin{cases} \pm Z_{\lambda}^{ \pm} \sqrt{|\ell|+1} & \text { for } \ell^{\prime}=-\ell \mp 1 \\ \mp Z_{\lambda}^{\mp} \sqrt{|\ell|} & \text { for } \ell^{\prime}=-\ell \pm 1 \\ (-1)^{\ell} & \text { for } \ell^{\prime}=-\ell \\ 0 & \text { otherwise }\end{cases}
$$

We see that (in our first-order approximation, see Eq. 6) these coefficients couple "neighboring" OAM modes with $\ell^{\prime}=-\ell \pm 1$, where the minus sign stems from image reversal upon reflection. In other words, the OAM spectrum acquires sidebands upon reflection. The complex-valued parameters

$$
Z_{\lambda}^{ \pm}=\frac{\theta_{0}}{2^{3 / 2}}(-1)^{\ell}\left(X_{\lambda} \pm i Y_{\lambda}\right)
$$

combine all dimensionless shifts. The intensity which appears in a specific sideband after reflection is $C_{\ell, \ell^{\prime}}^{\lambda}=\left|c_{\ell, \ell^{\prime}}^{\lambda}\right|^{2}$; it depends on the strength of the shifts via Eq. 9 . further it is proportional to $\ell$ and to the square of the beam opening angle $\theta_{0}^{2}$. In contrast to the previous case of beam shifts (Eq. 5), the OAM sideband method does not discriminate real (spatial) and imaginary (angular) components of the dimensionless shifts $X_{\lambda}$ and $Y_{\lambda}$, which is surprising.

To demonstrate experimentally the appearance of OAM sidebands under total internal reflection, we check these dependencies by varying $\ell$ and $\theta_{0}$. The setup is shown in Fig. 3, where we use again total internal reflection in a $45^{\circ}-90^{\circ}-45^{\circ}$ prism (BK7). We collimate a fibercoupled $635 \mathrm{~nm}$ diode laser with a $20 \times$ objective, then use a spatial light modulator to imprint the incoming-beam helical phase $\exp (i \ell \phi)$. This method produces, in terms of Laguerre-Gauss modes, a superposition of modes with the same azimuthal index $\ell$, but with many radial modes of different $p$; this superposition depends on the magnitude of $\ell$. This OAM beam is then reflected internally at the prism hypothenuse. We analyze the reflected beam by its OAM spectrum with another combination of SLM and a single-mode fiber, which in turn is connected to a photo diode. We align the setup for best mode matching between the single mode fibers of the laser and the detector for the case of $\ell=\ell^{\prime}=0$. To vary $\theta_{0}$, we introduce two microscope objectives $(10 \times, 0.25 \mathrm{NA}$, underfilled aperture). To compensate for small residual alignment errors, we use polarization modulation by $\lambda / 2$ wave plates on rotation stages, and determine the total polarization-differential OAM sideband intensity $I_{p d}(\ell)$, see [14]. To obtain a theoretical prediction, we use numerical modeling of the experiment: This is required because (i) our setup has a transmission which depends strongly on the selected OAM mode (i.e., transmission for $\ell^{\prime} \equiv \ell$, see supplementary information [14]); and (ii) 


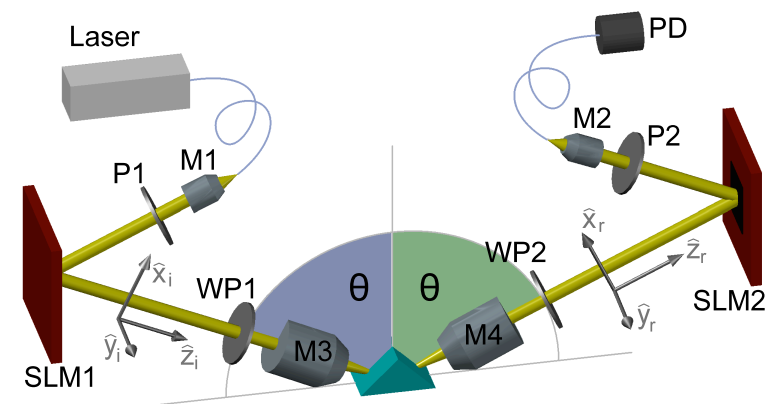

Figure 3. Experimental setup to measure the spatial Fresnel coefficients. We prepare the test beam using a fiber collimator (M1), polarizing optics (P1, WP1), and a spatial light modulator (SLM1). The beam is reflected internally at the prism hypothenus, and focussing and recollimation can be done with M3 and M4 to alter $\theta_{0}$. The analysis part consists of P2, WP2, SLM2, and M2; the transmitted intensity is measured using a photo-diode (PD).
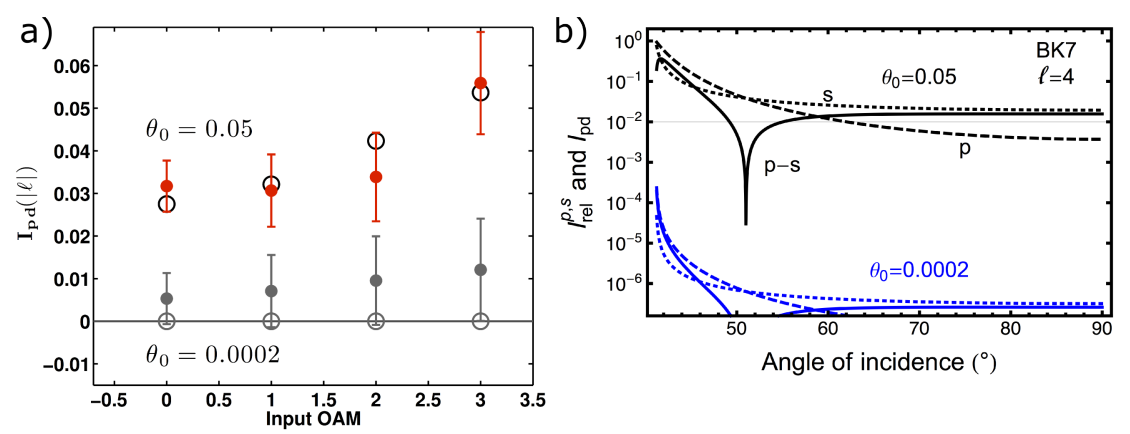

Figure 4. (a): Measurement of the spatial Fresnel coefficients under total internal reflection. Shown is the polarization-differential OAM sideband strength $I_{p d}(\ell)$ as a function of the input OAM $\ell$, for a collimated $\left(\theta_{0}=0.0002\right)$ and a focussed $\left(\theta_{0}=0.05\right)$ beam (filled circles with error bars). The theoretical prediction (open circles) is based upon numerical simulation of the experiment. (b): Exact theory for the OAM sideband intensity $I_{p d}(\ell)$ for $\ell=4$ and the full TIR range of angles of incidence, for true LG modes with $p=p^{\prime}=0$. Also shown are the relative sideband intensities $I_{r e l}^{p}$ and $I_{r e l}^{s}$, for $p$ and $s$ polarization, respectively, for both choices of $\theta_{0}$. We conclude that the OAM sidebands depend on $\ell$ and on $\theta_{0}$; and that already for mildly focussed beams the OAM sideband strength exceeds $1 \%$ over a large range of angles of incidence.

the radial-mode superposition as produced by the SLMs has to be taken into account. In any case, the OAM part of the modes is well defined throughout our setup.

In Fig. 4(a) we compare the OAM sideband intensity from experiment and theory at an angle of $45^{\circ}$, for a collimated $\left(\theta_{0}=0.0002\right)$ and a focussed $\left(\theta_{0}=0.05\right)$ beam. We can confirm that the sideband intensity depends on the input OAM $\ell$ and on the beam opening angle $\theta_{0}$, and agrees with the simulated data. Fig. 4(b) shows the theoretically calculated total sideband intensity for an incoming $\ell=4$ OAM beam over a larger range of incident angles. In the case of a focussed beam $\left(\theta_{0}=0.05\right)$, the sideband intensity exceeds $1 \%$ for $s$-polarization over the whole range of total internal reflection. This has to be taken into account if total internal reflection is used in, e.g., beam steering applications. 


\section{Discussion: OAM beam shifts and sidebands}

We compare now our two experiments: In the first case, we found that in total internal reflection, beam shifts are independent of the OAM of the beam, and independent of beam focussing. However, if we measure the OAM sidebands appearing during total reflection, both properties influence the result (i.e., the sideband strength). As is obvious from the similarity of the experiments, both effects describe the same underlying physical phenomenon: Diffractive corrections to geometric optics for total internal reflection. We want to explore this relation briefly theoretically. We start by writing the spatial part (for polarization $\lambda$ ) of the reflected field in a quantum-like notation based on the spatial Fresnel coefficients (we use their full form, see [14], for $\ell>0$ ):

$$
\begin{aligned}
\mid \text { out }\rangle_{\lambda}= & \sum_{\ell^{\prime}, p^{\prime}} a_{\lambda} r_{\lambda} c_{\ell, \ell^{\prime}, p, p^{\prime}}^{\lambda}|\lambda\rangle\left|\ell^{\prime}, p^{\prime}\right\rangle=a_{\lambda}(-1)^{\ell}|-\ell, p\rangle \\
& +a_{\lambda} Z_{\lambda}^{+} \sqrt{\ell+p+1}|-\ell-1, p\rangle+a_{\lambda} Z_{\lambda}^{+} \sqrt{p}|-\ell-1, p-1\rangle \\
& -a_{\lambda} Z_{\lambda}^{-} \sqrt{\ell+p}|-\ell+1, p\rangle-a_{\lambda} Z_{\lambda}^{-} \sqrt{p+1}|-\ell+1, p+1\rangle
\end{aligned}
$$

The real space representation of this leads to the centroid as follows:

$$
\langle\mathbf{R}\rangle(z)=\frac{\langle\text { out }|\mathbf{R}| \text { out }\rangle}{\langle\text { out }| \text { out }\rangle}
$$

To be able to compare this to Eq. 5. we need to find the sidebands for pure azimuthal LG input modes with $p=0$. Eq. 11 can easily be evaluated by using the known orthogonality relations of the LG modes, and by recognizing that the position operator $\mathbf{R}$ can be written as

$$
\mathbf{R}=r\left(\hat{\mathbf{x}}_{1}^{r} \cos \phi+\hat{\mathbf{x}}_{2}^{r} \sin \phi\right)=\frac{r}{2}\left[e^{i \phi}\left(\hat{\mathbf{x}}_{1}^{r}-i \hat{\mathbf{x}}_{2}^{r}\right)+e^{-i \phi}\left(\hat{\mathbf{x}}_{1}^{r}+i \hat{\mathbf{x}}_{2}^{r}\right)\right]
$$

We see that this operator couples modes with $\Delta \ell= \pm 1$ in $\langle$ out $|\mathbf{R}|$ out $\rangle$. Straight forward evaluation of Eq. 11 leads then exactly to Eq. 5 i.e., the dependency of $\ell$ and $\theta_{0}$ (which was implicit in the coefficients $\left.c_{\ell, \ell^{\prime}, p, p^{\prime}}^{\lambda}\right)$ disappears for total internal reflection.

Can we give an intuitive explanation why $\theta_{0}$-dependent OAM sidebands occur for TIR, while angular beam shifts are absent? In short, the measurement method is different. Spatial beam shifts are absolute, they do not depend on the beam waist. The OAM spectrum of a displaced beam, however, depends on the ratio of the displacement to the beam waist [16, 17]. If the beam is collimated, the Goos-Hänchen and Imbert-Fedorov shifts are usually very small compared to the beam waist, and OAM spectrum is not modified; if the beam is focussed, appreciable sidebands appear, in agreement with our experimental results in Fig. 栜.

In conclusion, we have studied the behavior of OAM beams under total internal reflection. We have investigated this by two complementary methods: Firstly, by the analysis of optical beam shifts, and secondly by the observation of modifications in the OAM spectrum of a probe beam by the spatial Fresnel coefficients. In the first case, we found that OAM does not modify the spatial beam shifts under total reflection. In the other case, the opposite is true: The OAM spectrum of a beam is modified under TIR, and the strength of these modifications increase with the OAM of the beam as well as its focussing. To resolve this issue, we have shown how to derive the beam shifts from the spatial Fresnel coefficients in the short didactical discussion at the top of this section.

Very recently, a third method to study such diffractive corrections was found: It turns out that physical reflection induces a split-up of a high-order vortex into spatially separated first order 
vortices, and the splitting is characteristic for a given experimental condition [18. Based upon our experimental result here, we would expect that such split-up will occur also under TIR: Vortex splitting can be explained by coherent background fields [19], and the OAM sidebands, as found here, are of course an example of such a coherent background field.

\section{Acknowledgments}

We acknowledge financial support by NWO and the EU STREP program 255914 (PHORBITECH).

\section{References}

[1] F. Goos and H. Hänchen. Ein neuer und fundamentaler Versuch zur Totalreflexion. Ann. Phys., 436:333-346, 1947. doi:10.1002/andp.19474360704

[2] F. I. Fedorov. On the theory of total internal reflection. Dokl. Akad. Nauk SSSR, 105:465, 1955.

[3] Christian Imbert. Calculation and experimental proof of the transverse shift induced by total internal reflection of a circularly polarized light beam. Phys. Rev. D, 5(4):787-796, Feb 1972. doi:10.1103/PhysRevD.5.787

[4] Konstantin Yu. Bliokh and Yury P. Bliokh. Conservation of angular momentum, transverse shift, and spin hall effect in reflection and refraction of an electromagnetic wave packet. Phys. Rev. Lett., 96(7):073903, 2006. doi:10.1103/PhysRevLett.96.073903.

[5] Onur Hosten and Paul Kwiat. Observation of the Spin Hall Effect of Light via Weak Measurements. Science, 319(5864):787-790, 2008. doi:10.1126/science.1152697.

[6] Andrea Aiello, Norbert Lindlein, Christoph Marquardt, and Gerd Leuchs. Transverse angular momentum and geometric spin hall effect of light. Phys. Rev. Lett., 103(10):100401, 2009. doi:10.1103/PhysRevLett.103.100401.

[7] C. Chiu Chan and T. Tamir. Angular shift of a gaussian beam reflected near the brewster angle. Opt. Lett., 10(8):378-380, 1985.

[8] A. Aiello and J. P. Woerdman. Role of beam propagation in Goos-Hänchen and ImbertFedorov shifts. Opt. Lett., 33(13):1437-1439, 2008.

[9] M. Merano, A. Aiello, M. P. van Exter, and J. P. Woerdman. Observing angular deviations in the specular reflection of a light beam. Nat. Photon., 3(6):337-340, jun 2009.

[10] C. W. J. Beenakker, R. A. Sepkhanov, A. R. Akhmerov, and J. Tworzydło. Quantum gooshänchen effect in graphene. Phys. Rev. Lett., 102(14):146804, Apr 2009. doi:10.1103/ PhysRevLett.102.146804.

[11] Victor-O. de Haan, Jeroen Plomp, Theo M. Rekveldt, Wicher H. Kraan, Ad A. van Well, Robert M. Dalgliesh, and Sean Langridge. Observation of the goos-hänchen shift with neutrons. Phys. Rev. Lett., 104(1):010401, Jan 2010. doi:10.1103/PhysRevLett.104.010401.

[12] M. Merano, N. Hermosa, J. P. Woerdman, and A. Aiello. How orbital angular momentum affects beam shifts in optical reflection. Phys. Rev. A, 82(2):023817, Aug 2010. doi: 10.1103/PhysRevA.82.023817

[13] L. Allen, M. W. Beijersbergen, R. J. C. Spreeuw, and J. P. Woerdman. Orbital angular momentum of light and the transformation of laguerre-gaussian laser modes. Phys. Rev. A, 45(11):8185-8189, Jun 1992. doi:10.1103/PhysRevA.45.8185. 
[14] W. Löffler, Andrea Aiello, and J. P. Woerdman. Observation of oam sidebands due to optical reflection. pre-print, apr 2012. arXiv:1204.4003.

[15] Andrea Aiello. Goos-hänchen and imbert-fedorov shifts: a novel perspective. New J. Phys., 14(1):013058, 2012.

[16] Graham Gibson, Johannes Courtial, Miles Padgett, Mikhail Vasnetsov, Valeriy Pas'ko, Stephen Barnett, and Sonja Franke-Arnold. Free-space information transfer using light beams carrying orbital angular momentum. Opt. Express, 12(22):5448-5456, 2004.

[17] M V Vasnetsov, V A Pas'ko, and M S Soskin. Analysis of orbital angular momentum of a misaligned optical beam. New J. Phys., 7(1):46, 2005.

[18] M.R. Dennis and J.B. Götte. Topological aberration of optical vortex beams and singularimetry of dielectric interfaces. pre-print, may 2012. arXiv:1205.6457.

[19] Vladimir G. Denisenko, Alexander Minovich, Anton S. Desyatnikov, Wieslaw Krolikowski, Marat S. Soskin, and Yuri S. Kivshar. Mapping phases of singular scalar light fields. Opt. Lett., 33(1):89-91, Jan 2008. doi:10.1364/0L.33.000089. 